164 embolization procedures including two peri/post procedural hemorrhages, one dissection, and one infarct. All patients undergoing microsurgical resection had a complete cure on post-operative angiography. Immediate postoperative mRS and long term follow up mRS (mean of 55 months) was determined after curative treatment. Good long term outcomes (mRS $\leq 2)$ was seen in $95 \%$ of unruptured AVM patients and 92\% of ruptured AVM patients postoperatively. Transient and permanent neurological deficits were seen in 5\% and $1 \%$ of patients after curative treatment.

Conclusions Multimodal therapy of low grade AVMs can be performed with low morbidity and high cure rates in high volume centers. By comparison with other published studies, our outcomes suggest that pre-operative embolization is a safe adjunct to definitive curative treatment.

Disclosures A. Wang: None. E. Connolly: None. R. Solomon: None. S. Lavine: None. P. Meyers: None.

\section{E-071 PERCUTANEOUS ALCOHOL EMBOLIZATION OF SYMPTOMATIC VERTEBRAL HEMANGIOMA USING COLUMN TECHNIQUE: A CASE REPORT}

S Male*, T Mehta, A Khan, A Grande, R Tummala, B Jagadeesan. University of Minnesota, Minneapolis, MN

\subsection{6/neurintsurg-2019-SNIS. 146}

Background Hemangiomas are the most common vertebral body tumors typically discovered as incidental findings on spinal imaging. In $1 \%$ of patients, hemangiomas can be symptomatic causing persistent back pain that necessitate treatment. Percutaneous alcohol embolization (PAE) is an effective but challenging way of treating these lesions. We hereby report a case of vertebral hemangioma treated with PAE with unique technique.

Methods 59-year-old man with chronic back pain refractory to medications, physical therapy and chiropractic maneuvers presented to our clinic. His MRI spine revealed multiple vertebral hemangiomas, most prominent at T8 and T10. On examination, the point of maximum tenderness was at T10. Limited spinal angiogram revealed tumor blush at the sites of vertebral hemangiomas. Under sterile conditions, two 10-gauge needles were advanced in the T10 vertebral body via transpedicular approach. The contrast injection revealed tumor blush and venous outflow into the azygous system. For PAE, we formed columns of contrast-absolute ethanol-contrast to avoid alcohol dilution and for adequate visualization during embolization. (figure 1) A bubble trapped between these columns, initially

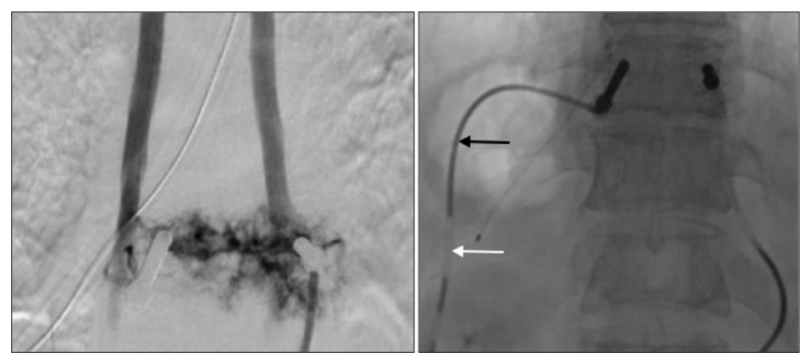

Abstract E-071 Figure 1 inadvertently and later intentional, also helped to keep the columns separate. The mixing of contrast to absolute ethanol is an alternative to this technique. However, it could decrease the efficacy of absolute ethanol in obliteration of hemangioma. Patient tolerated the procedure very well and endorsed clinical improvement on day 1.

Conclusion During PAE for vertebral hemangiomas, formation of columns of contrast and absolute ethanol during embolization is an effective technique. It helps to preserve higher alcohol concentration and in turn, efficacy of embolization while maintaining adequate visualization.

Disclosures S. Male: None. T. Mehta: None. A. Khan: None. A. Grande: None. R. Tummala: None. B. Jagadeesan: None.

\section{E-072 IMPACT OF OBESITY ON SHORT-TERM IN-HOSPITAL OUTCOMES IN PATIENTS UNDERGOING SPINAL FUSION PROCEDURES}

T Colburn*, D Schirmer, Z Rose-Reneau, B Wright. Kansas City University of Medicine and Biosciences, Kansas City, MO

10.1136/neurintsurg-2019-SNIS.147

Background Spinal fusion is a surgical procedure utilized to treat or alleviate several spinal diagnoses including: tumor, spinal stenosis, degenerative disc disease, scoliosis, and spondylolisthesis. Previous studies have explored clinical outcomes for this intervention. This study aims to further investigate shortterm outcomes of patients after spinal fusion by looking at the impact of obesity.

Methods This retrospective cohort study utilized data from the Nationwide Inpatient Sample (NIS) to identify adult patients $(18+)$ from 2012-2015 who underwent spinal fusion. ICD-9 codes identified these patients; specifically patients diagnosed with obesity. Any patients missing important clinical identifiers (age, gender, cause of death) and patients without spinal fusion intervention were excluded. Data analyses assessed hospital length of stay (LOS), inpatient charges, average age of admission and mortality rate.

Results Of the 290,752 patients that underwent spinal fusion procedures, 374 had the diagnosis of obesity $(\mathrm{OB})$ :

Mean mortality rate of patients was significantly increased (1.9\%, OB vs. $0.6 \%$, no OB $\mathrm{p}=0.01)$

LOS in patients with obesity who underwent spinal fusion was significantly increased (7.02 days, OB vs. 4.02 days, no $\mathrm{OB}, \mathrm{p}=0.0001)$

Total hospital charges were significantly increased $(\$ 142,853.68$, OB vs. $\$ 98,294.43$, no OB, $p=0.0001)$

Age at admission was not significant in patients with obesity (58.43 years, OB vs. 57.69 years, no OB, $\mathrm{p}=.307$ )

Conclusion Patients who are obese and undergo a spinal fusion procedure suffer from increased mortality rate, LOS, and total hospital charges. This study aims to provide physicians with information in the management of patients with obesity that undergoes spinal fusion procedures. Peri-procedural patient optimization could provide a potential avenue to lower LOS, total in-hospital charges, and mortality in patients. Disclosures T. Colburn: None. D. Schirmer: None. Z. RoseReneau: None. B. Wright: None. 